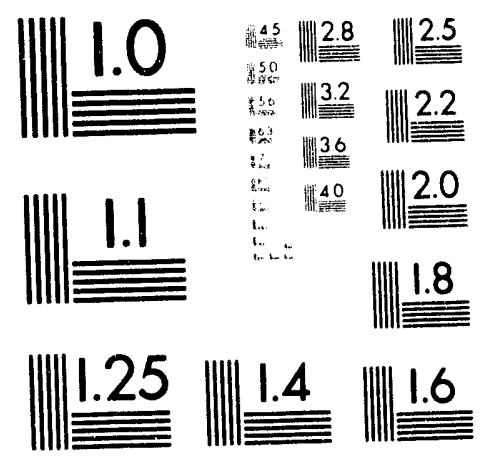



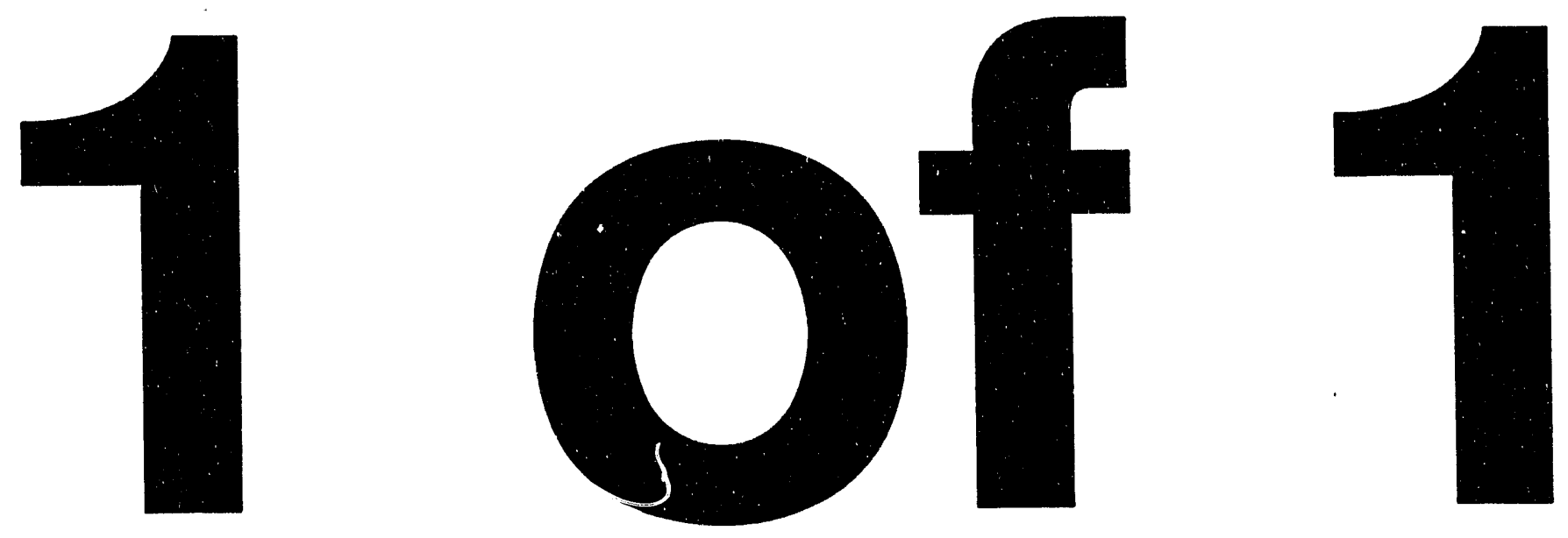
August 21, 1992

Bruce A. Gold

MR 2B-C

\section{AFBC ROADBED PROJECT GROUNDWATER DATA}

TVA permitted the use of AFIS material in a section of roadbed at Paducah, Kentucky, for the purpose of demonstrating its usability as a roadbed base. To determine if the material would leach and contaminate groundwater, four wells and seven lysimeters were installed beside and in the roadbed base material.

In August 1991, TVA Field Engineering visited the AFBC Roadbed Project to collect samples and water quality data. The goal was to collect samples and data from four wells and seven lysimeters. All atteinpts to collect samples from the lysimeters failed with one exemption. All attempts to collect samples from the groundwater wells were successful.

Attempts were made to sample all seven lysimeters. An apparatus consisting of a vacuum tank, a vacuum gauge, and a filtering flask was attached to each of four lysimeters. The other three had only a filtering flask attached. Vacuums were applied to all seven systems at approximately $8 \mathrm{p} \cdot \mathrm{m}$. On August 8 . None of the lysineters had accumulated any moisture by $3 \mathrm{p} . \mathrm{m}$. on August 9 . However, one tubular lysimeter located at box one did later accumulate enough water to sample.

A diligent effort was made to make the lysimeters function. Several attempts were made to collect the samples and/or ascertain the lysimeters' conditions. For example, each of the tubular lysimeters ( $t$ wo-line type) were tested for their ability to hold a vacuum. The plate lysimeters were also tested for their ability to hold a vacuum. Attachment 1 summarizes the findings about each of the lysimeters and provides the moisture readings associated with each lysimeter.

The groundwater well sampling was successful. All four wells were high producing wells and presented no problem in sampling. The attached groundwater quality data field worksheets show all the requested field data. Also, Attachment 2 displays the location of each well and its relative elevation. Please note that ali water surface measurements were made from the top of the metal casing.

The analytical data from the four wells and one lysimeter are also attached.

The well data is typical of groundwater in the Paducah, Kentucky area indicating that it was not affected by the AFBC roadbed material. The analysis of the lysimeter shows concentrations for iron and manganese above normal background levels, however, the data do not reflect significant concentrations of these heavy metals. Also, the difficulty in obtaining the lysimeter samples and the fact that the samples had to be composited to obtain sufficient quantity to analyze would make a qualitative evaluation of the data questionable. 
Bruce A. Gold

Page 2

August 21, 1992

If you have any questions regarding this information, please call me at
(615) $632-1883$.

Wally Carpenter

Engineering Laboratory

$L A B \quad 1 A-N$

WGC : CP

Attachments

cc: Files, LAB $1 \mathrm{~A}-\mathrm{N}$

$3023 c$

\section{DISCLAIMER}

This report was prepared as an account of work sponsored by an agency of the United States Government. Neither the United States Government nor any agency thereof, nor any of their employees, makes any warranty, express or implied, or assumes any legal liability or responsibility for the accuracy, completeness, or usefulness of any information, apparatus, product, or process disclosed, or represents that its use would not infringe privateiy owned rights. Reference herein to any specific commercial product, process, or service by trade name, trademark, manufacturer, or otherwise does not necessarily constitute or imply its endorsement, recommendation, or favoring by the United States Government or any agency thereof. The views and opinions of authors expressed herein do not necessarily state or reflect those of the United States Government or any agency thereof. 


\author{
Attachment 1 \\ AFBC Roadbed Project - Paducah, Kentucky \\ Condition of Lysimeters
}

\section{Lysimeter}

Box 1 Tubular

Box 2 Plate

Box 2 Tubular

Box 3 Plate

Box 3 Tubular

Box 4 Plate

Box 4 Tubular
Will hold

vacuum?

$$
\begin{array}{r}
\text { Yes } \\
\text { No } \\
\text { Yes } \\
\text { No } \\
\text { No } \\
\text { No } \\
\text { Yes }
\end{array}
$$

Will allow

air passage?

* Yes

---

No

--

Yes

-.-

No
Moisture

Content (\%)

97

7

2

11

9

1

0

* The air passage test was used to see if air forced in one line of would return through the lysimeter's adjacent line. The air (or water sample) should return if the system is working. The absence of returning air indicates line blockage, line collapse, line perforation, or problems with the lysimeter itself. The plate lysimeters have only one line, therefore, the air passage test is not applicable.

LMK-78(3) 


\section{AFBC ROADBED PROJECT}

Bleich Road - Puducah, Kentucky

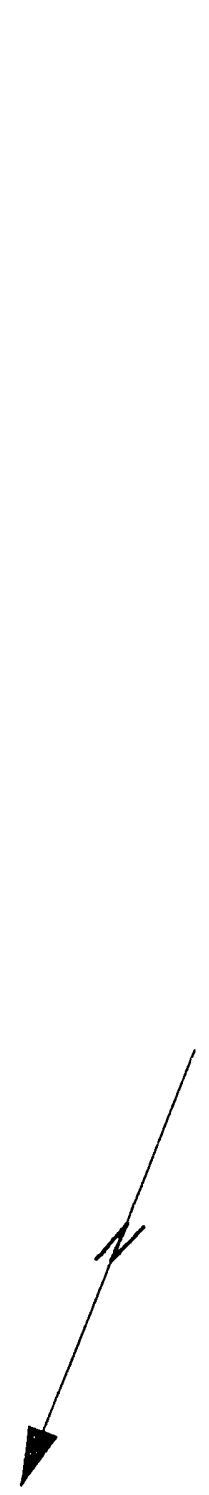

๓0 BOX 4

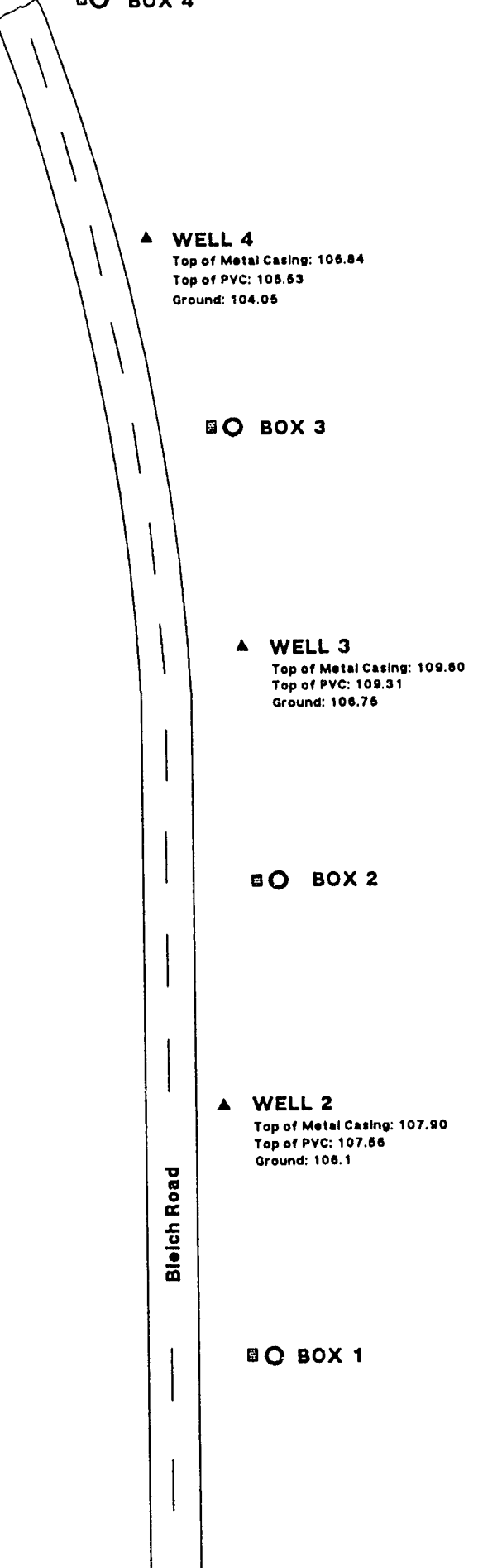

- WELL 1 Top of Motal Casing: 109.41 Top of PVC: 109.18

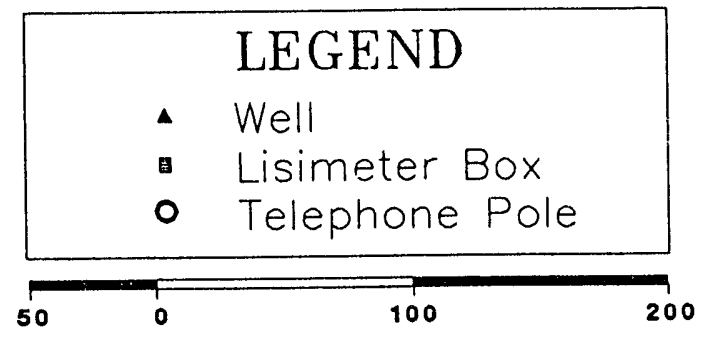




\section{Ground Water Quality Data Field Worksheet}

(Chemical Data)

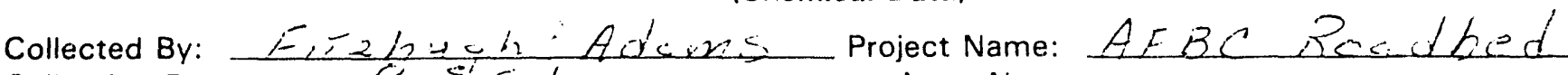

Collection Date: $8-9 ; 1$

Sample Tag No:: AE\&C-1e!

Collection Method: Bladder Pump_L_ Centrifugal Pump Acct. No.

Well ID:

Stabilization Test: Start Time: 125.5

W.S. Elevation:
Bailer

End Time:

W.S. Elevation:
Kemmerer

Description of RP:

\begin{tabular}{|c|c|c|c|c|c|c|c|}
\hline$\frac{\text { Time }}{(\min )}$ & $\frac{\text { Pump }}{\text { Rate }}$ & $\begin{array}{l}\begin{array}{l}\text { Water } \\
\text { Surface }\end{array} \\
\text { (ft) }\end{array}$ & $\frac{\text { Temperature }}{\left.{ }^{\circ} \mathrm{C}\right)}$ & $\mathrm{pH}$ & $\frac{D O}{(\mathrm{mg} / \mathrm{L})}$ & $\frac{\text { Conductivity }}{(\mu \mathrm{mhos} / \mathrm{cm})}$ & $\frac{\text { ORP }}{(m V)}$ \\
\hline 1255 & 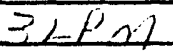 & 24.17 & 19.5 & 5.5 & 5.4 & 434 & 322 \\
\hline$\angle 3<\sigma$ & & & $1 x, 1$ & 55 & 4,3 & 550 & 325 \\
\hline $12<1-$ & & 24.2 & $18,: 2$ & 5.5 & 4.2 & 520 & $3 / 9$ \\
\hline $13 / 0$ & & & 18,2 & 52 & 40 & 562 & $2 / 2$ \\
\hline$i=i \dot{5}^{-}$ & & 21,0 & 18,2 & 5.5 & $4 \Omega$ & 543 & $2 ; 2$ \\
\hline & & & & & & & \\
\hline & & & & & & & \\
\hline & & & & & & & \\
\hline & & & & & & & \\
\hline & & & & & & & \\
\hline & & & & & & & \\
\hline & & & & & & & \\
\hline & & & & & & & \\
\hline & & & & & & & \\
\hline & & & & & & & \\
\hline & & & & & & & \\
\hline & & & & & & & \\
\hline & & & & & & & \\
\hline
\end{tabular}

Sample Field Measuremments (at time of sample collection):

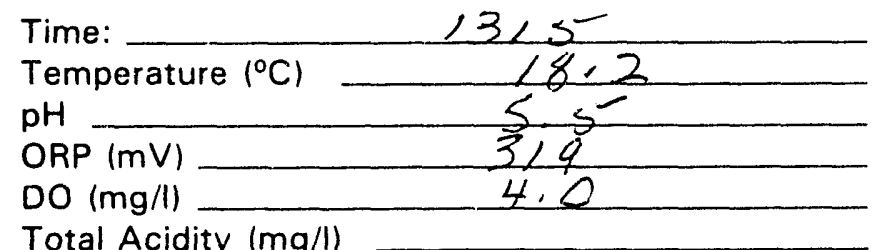

Total Acidity (mg/l)

Sample Turbidity: Clear
Slightly Turbid
Phenol Alkalinity $(\mathrm{mg} / \mathrm{l})$

Total Alkalinity $(\mathrm{mg} / \mathrm{l}) \quad 30$

Conductivity $(\mu \mathrm{mhos} / \mathrm{cm}) \quad 5 \mathrm{~kg}$

Odor $\quad \mu<\mu e$

Sample Color Misd $d y$ Turbid Highly Turbid L

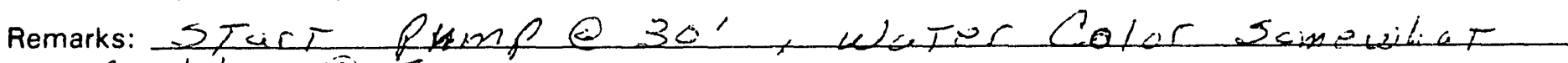

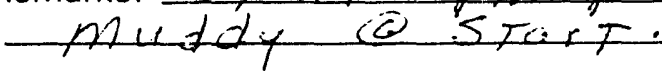

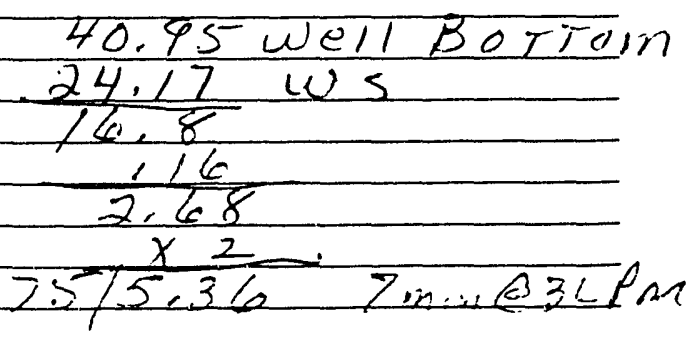

Volume of water in well casing:

Volume of purged well water:

Reviewed by

Date

TVA 30066A (S\&F OPS-11-87) 


\section{Ground Water Quality Data Field Worksheet}

\section{(Chemical Data)}

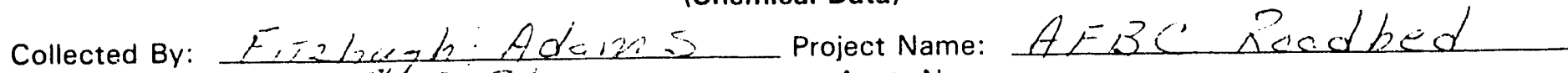

Collection Date: $8 \div 7.91$

Sample Tag No.: $4 E B C$ wi.2

Collection Method: Bladder Pump Acct. No.:

Well ID:

Centrifugal Pump

Stabilization Test: $\begin{aligned} \text { Start Time: } & 121.5 \\ & \text { W.S. Elevation: } \frac{22.1}{22.1}\end{aligned}$

$A F B C \omega z$ Bailer Kemmerer End Time: $\frac{123.5}{25.3}$

Description of RP:

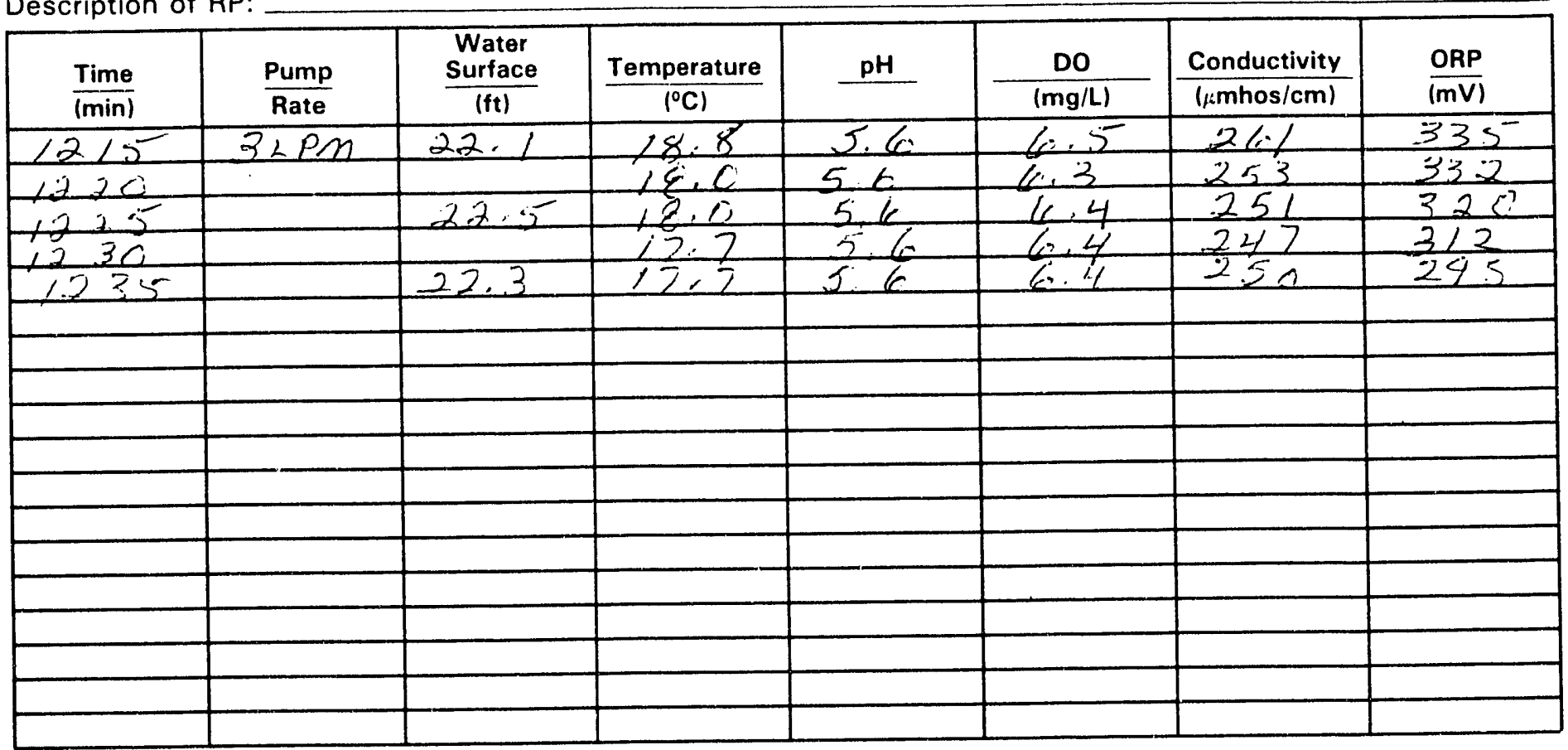

Sample Field Measuremments (at time of sample collection):

Time:

Temperature $\left({ }^{\circ} \mathrm{C}\right)$

$\mathrm{pH}$

ORP (mV)

DO (mg/l)

Total Acidity (mg/l)

Sample Turbidity: Clear
1235

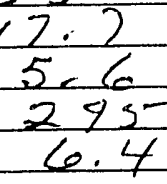

Phenol Alkalinity (mg/l)

Total Alkalinity (mg/l)

Conductivity $(\mu \mathrm{mhos} / \mathrm{cm})$

Odor

Aiciele

Sample Color Slighily Turbid Slightly Turbid 1 Turbid Highly Turbid

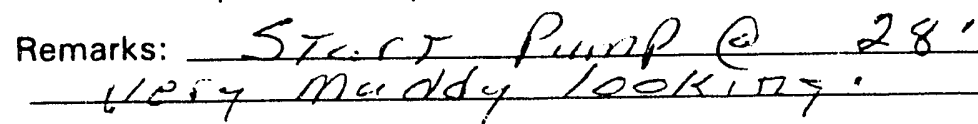
werer (e) STait wis

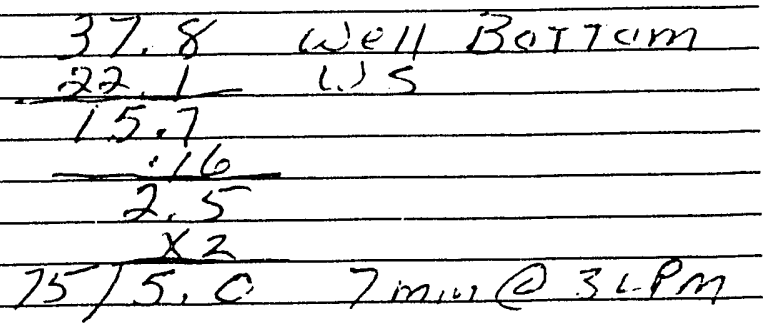

Volume of water in well casing:

Volume of purged well water:

Reviewed by

Date 


\section{Ground Water Quality Data Field Worksheet}

(Chemical Data)

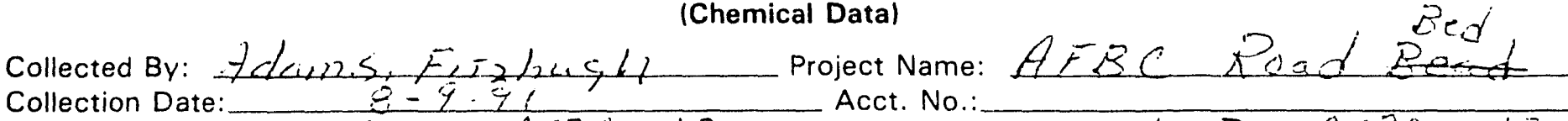

Sample Tag No.: Ne. 3 AFBC-Wi3 Well ID:

Collection Method: Bladder Pump_L Centrifugal Pump $\sim_{\text {Bailer }} \sim_{\text {Kemmerer }}^{\prime \prime}$

Stabilization Test: Start Time: OG/.5

W. Elevation: 25.9 End Time: 0940

Description of RP:

\begin{tabular}{|c|c|c|c|c|c|c|c|}
\hline$\frac{\text { Time }}{(\min )}$ & $\frac{\text { Pump }}{\text { Rate }}$ & $\begin{array}{l}\text { Water } \\
\text { Surface } \\
\frac{(\mathrm{ft})}{}\end{array}$ & $\frac{\text { Temperature }}{\left.{ }^{\circ} \mathrm{C}\right)}$ & $\mathrm{pH}$ & $\frac{\mathrm{DO}}{(\mathrm{mg} / \mathrm{L})}$ & $\frac{\text { Conductivity }}{(\mu \mathrm{mhos} / \mathrm{cm})}$ & $\frac{\text { ORP }}{(\mathrm{mV})}$ \\
\hline 1915 & $3 \angle P M$ & 25.9 & 110.9 & 5.6 & $\ln , 1$ & 98 & +306 \\
\hline 1423 & & & ilc.3 & 5.5 & 5.5 & 48 & \pm 320 \\
\hline 4025 & & & $i 1,1$ & 5,5 & 5.4 & $9: 2$ & +2.7 \\
\hline $0,43 c$ & & & $1 / \mathrm{s}, 1$ & 5.5 & 5.4 & 97 & $+22=$ \\
\hline C435 & & 272 & 16.0 & 5.5 & 5.4 & 97 & $+22 k$ \\
\hline 6440 & & 26.6 & LC. C & 5.5 & 54 & 97 & +3210 \\
\hline & & & & & & & \\
\hline & & & & & & & \\
\hline & & & & & & & \\
\hline & & & & & & & \\
\hline & & & & & & & \\
\hline & & & & & & & \\
\hline & & & & & & & \\
\hline & & & & & & & \\
\hline & & & & & & & \\
\hline & & & & & & & \\
\hline & & & & & & & \\
\hline & & & & & & & \\
\hline
\end{tabular}

Sample Field Measuremments (at time of sample collection):

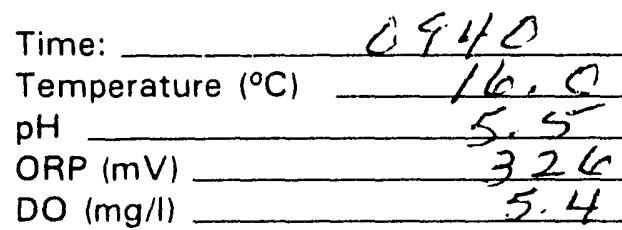

Total Acidity (mg/l)

Sample Turbidity: Clear
Slightly Turbid
Phenol Alkalinity $(\mathrm{mg} / \mathrm{l})$

Total Alkalinity $(\mathrm{mg} / \mathrm{l})$

Conductivity $(\mu \mathrm{mhos} / \mathrm{cm})$

Odor

Sample Color

4 cine

Turbid

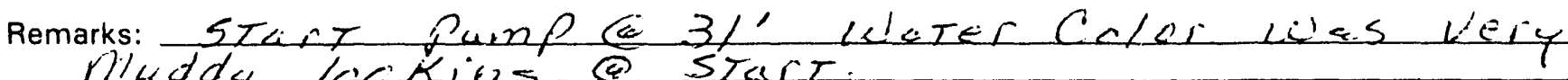
Iikddy lockik25 (a) STarT.

$$
\frac{\frac{38.2 .5}{25.90} \text { wiellBetion }}{\frac{12.35}{x \cdot 16}}
$$

Volume of water in well casing:

Volume of purged well water:

Reviewed by Date

TVA 30066A (S\&F OPS-11.87) 


\section{Ground Water Quality Data Field Worksheet}

(Chemical Data)

Collected By: Adams, Firzbre gla Project Name: AFBC Readbed

Collection Date:__ 8. $\quad$ _ -91

Sample Tag No.: AEBC $u^{\prime} \cdot 4$

Collection Method: Bladder Pump

Stabilization Test: Start Time:

W.S. Elevation: 20.3
Well ID: AFBC. - H 4

Centrifugal Pump

End Time: 1025

W.S. Elevation:

Description of RP:

\begin{tabular}{|c|c|c|c|c|c|c|c|}
\hline$\frac{\text { Time }}{(\min )}$ & $\frac{\text { Pump }}{\text { Rate }}$ & $\begin{array}{l}\text { Water } \\
\text { Surface } \\
\text { (ft) }\end{array}$ & $\frac{\text { Temperature }}{\left({ }^{\circ} \mathrm{C}\right)}$ & $\mathrm{pH}$ & $\frac{\mathrm{DO}}{(\mathrm{mg} / \mathrm{L})}$ & $\begin{array}{l}\text { Conductivity } \\
\mu \text { mhos } / \mathrm{cm}) \\
\end{array}$ & $\frac{\text { ORP }}{(\mathrm{mV})}$ \\
\hline$\angle C^{\circ}: 0$ & $3 L P M$ & $2 x, 3$ & ils. 7 & 56 & 7.5 & 144 & +340 \\
\hline $1105^{2}$ & & & $\angle 1.4$ & 5.7 & 7,5 & 101 & +330 \\
\hline 1010 & & 21.0 & 11.8 & 56 & 2.5 & 100 & $+32=$ \\
\hline $1(1) 5$ & & & 11.7 & 5.6 & $7,5=$ & $\varepsilon \varepsilon$ & +331 \\
\hline 1470 & & 20.4 & 16.7 & 5.6 & 7.5 & 97 & 331 \\
\hline \multirow{2}{*}{\multicolumn{8}{|c|}{$1 i 25$}} \\
\hline & & & & & & & \\
\hline & & & & & & & \\
\hline & & & & & & & \\
\hline & & & & & & & \\
\hline & & & & & & & \\
\hline & & & & & & & \\
\hline & & & & & & & \\
\hline & & & & & & & \\
\hline & & & & & & & \\
\hline 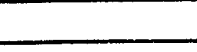 & & & & & & & \\
\hline & & & & & & & \\
\hline
\end{tabular}

Sample Field Measuremments (at time of sample collection):

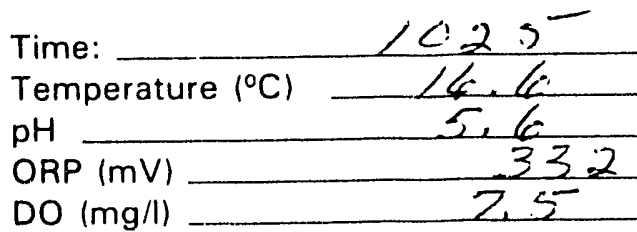

Total Acidity (mg/l)

Sample Turbidity: Clear_i Slightly Turbid

Remarks: STerT Pdmp C $20^{\prime}$

Siar 5 .
Phenol Alkalinity $(\mathrm{mg} / \mathrm{l})$

Total Alkalinity $(\mathrm{mg} / \mathrm{l})-26$

Conductivity $(\mu \mathrm{mhos} / \mathrm{cm})$ qL

Odor la cue

Sample Color leipar Turbid Highly Turbid

uiser Colur meddy (2)

starer.

Volume of water in well casing

TVA 30066A (S\&F OPS-11-87) 


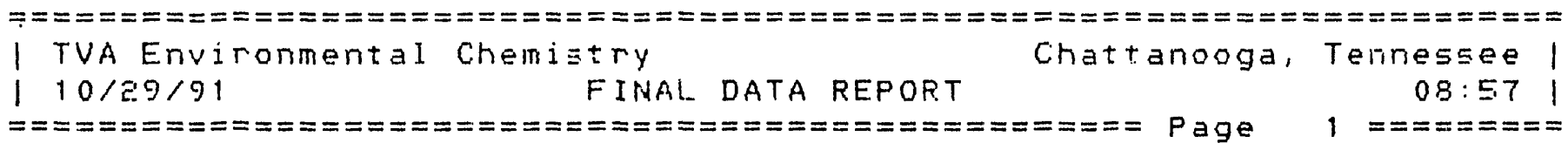

Lab Sample Number: $: 91 / 15450$

Project Leader : Gilberto Quintero

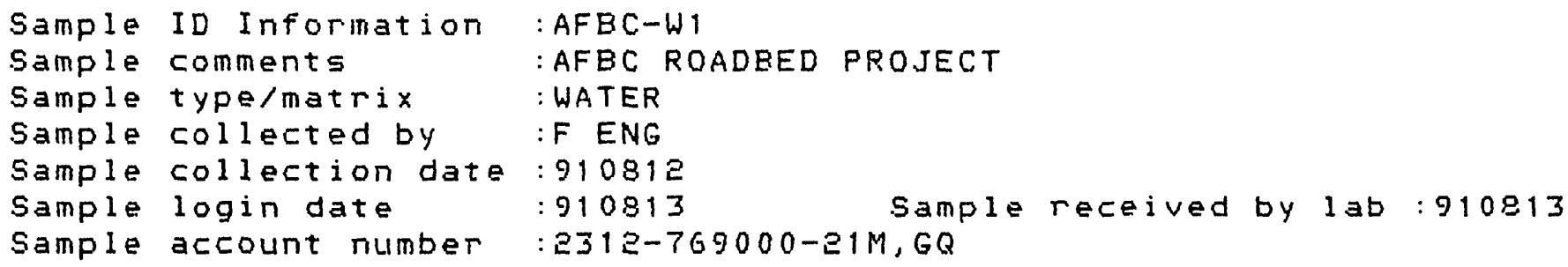

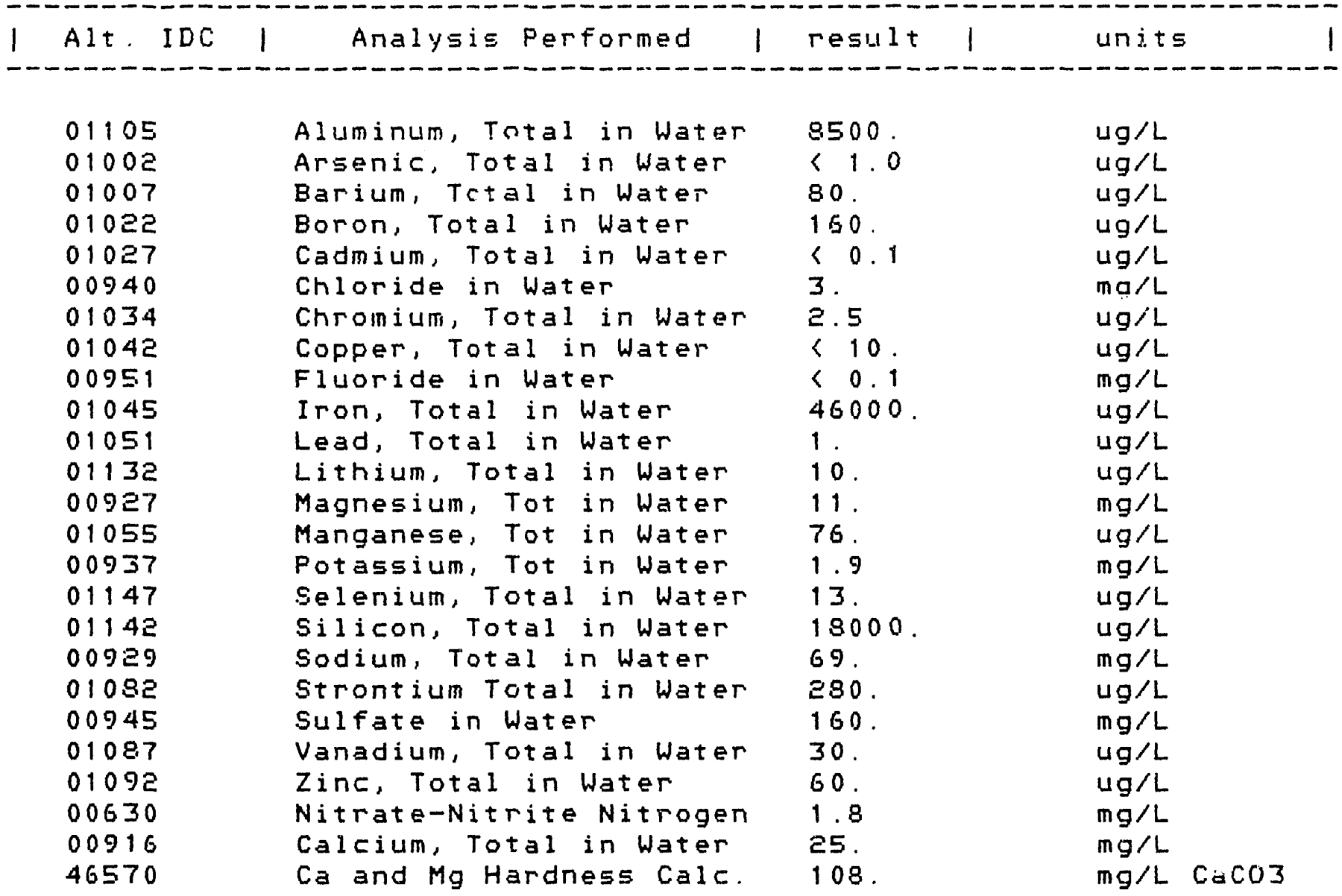




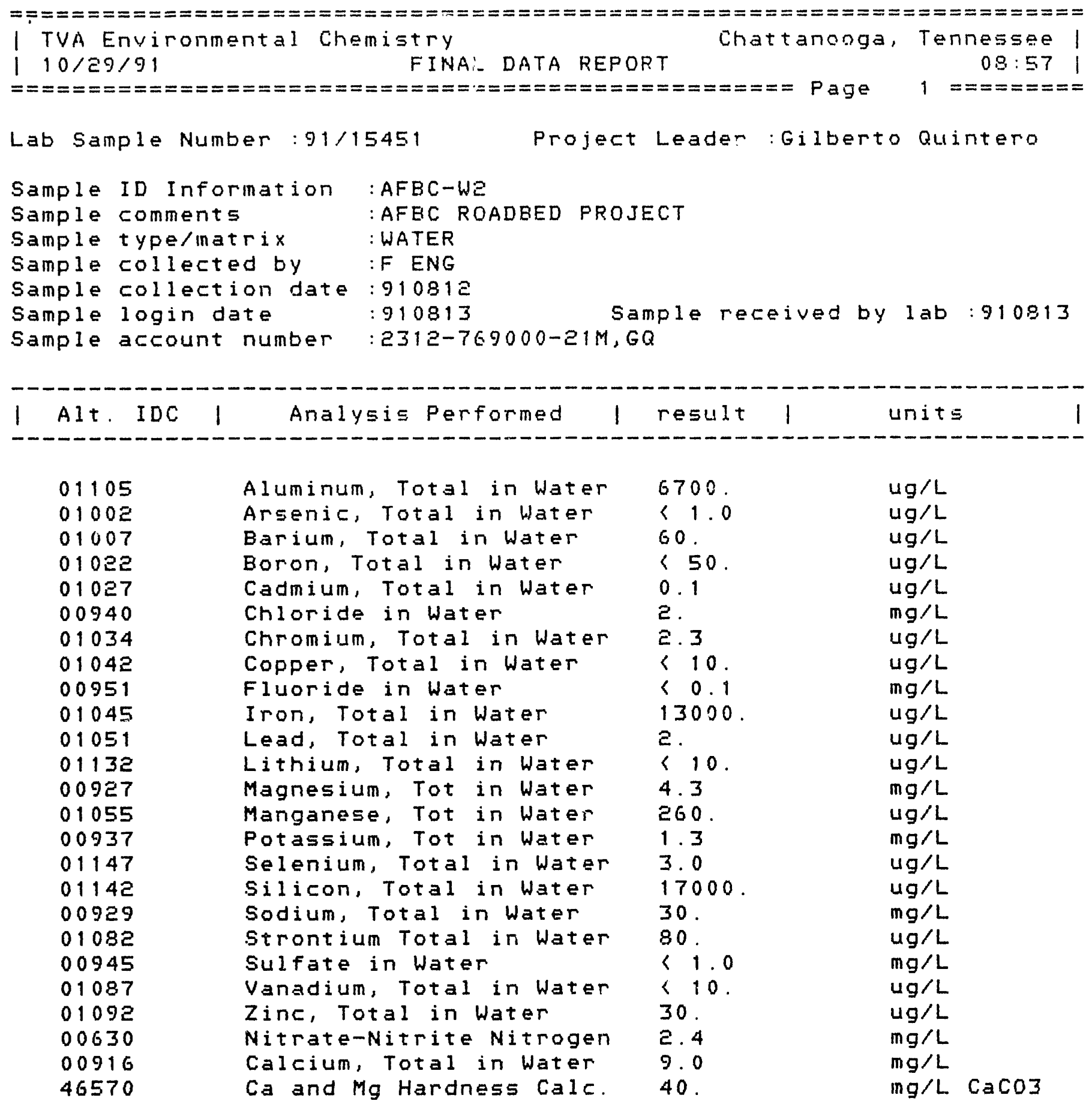




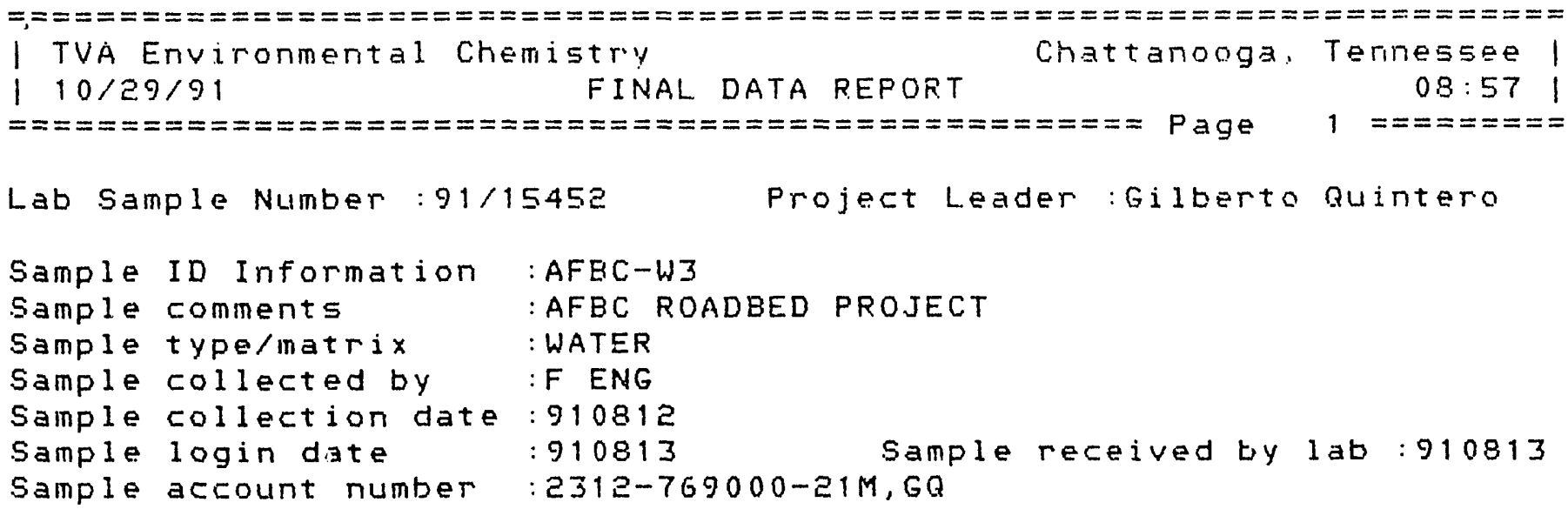

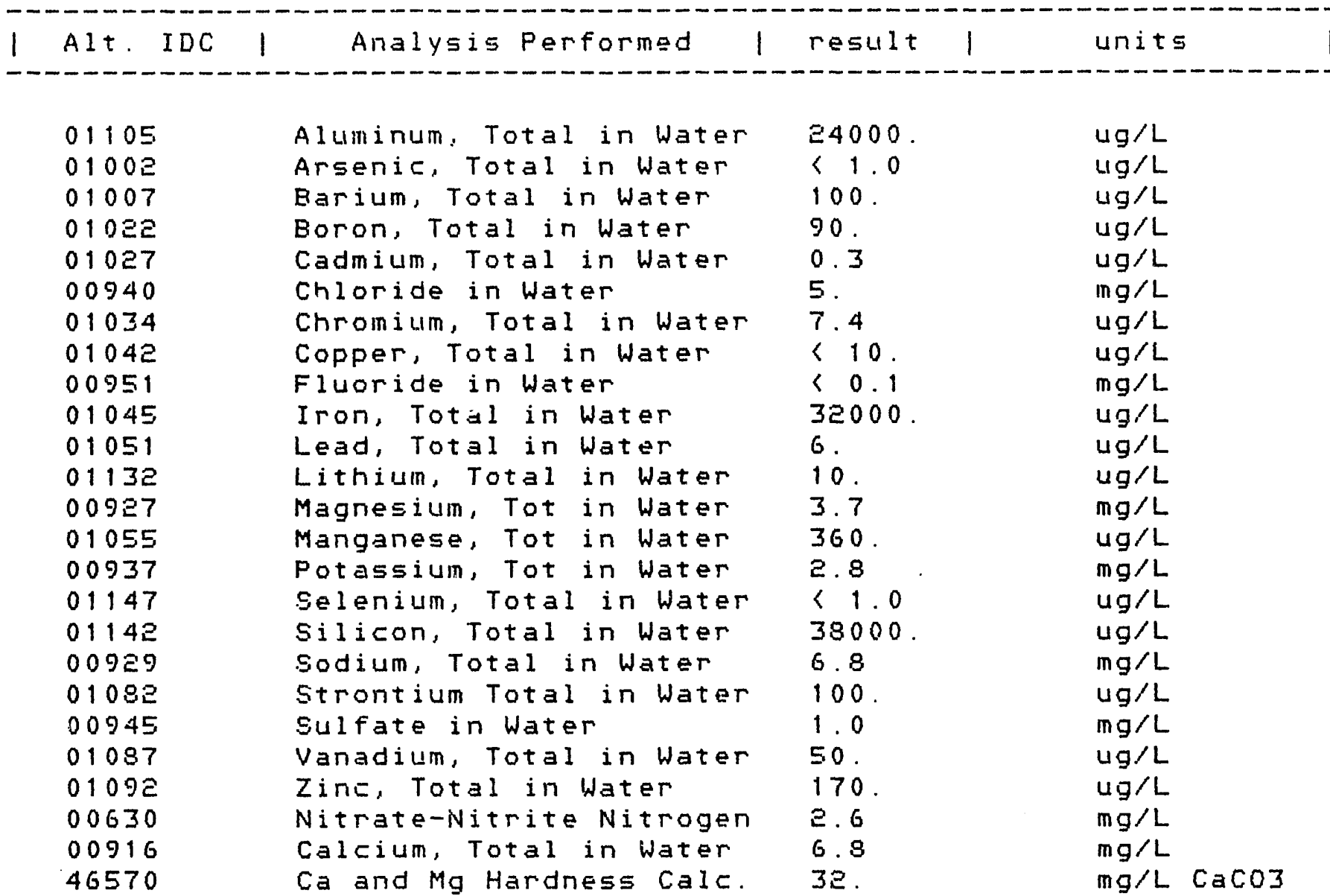




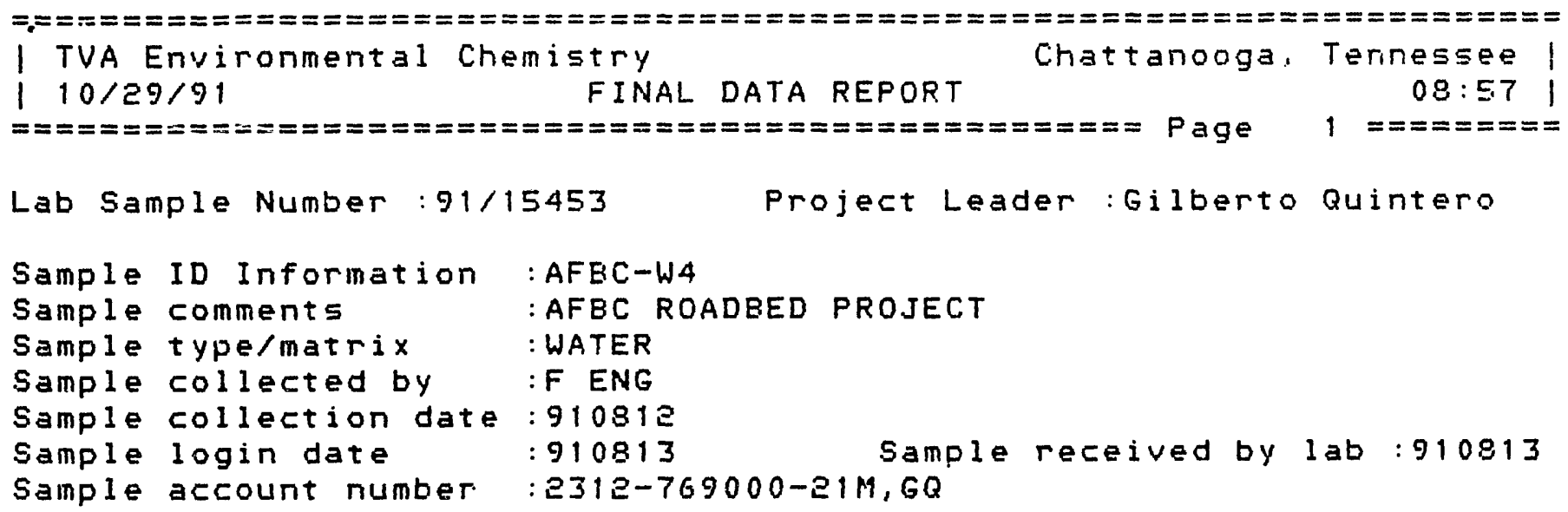

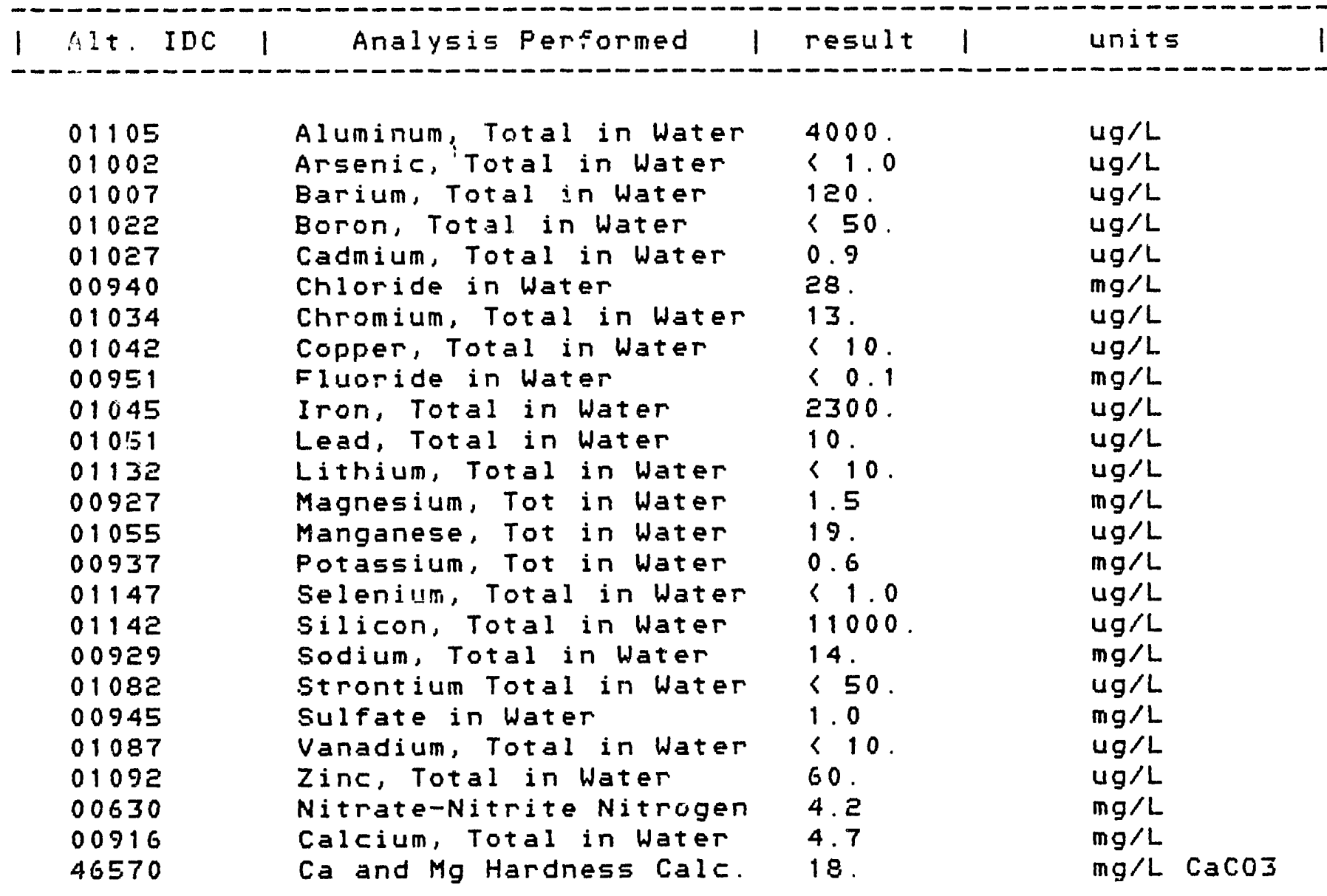




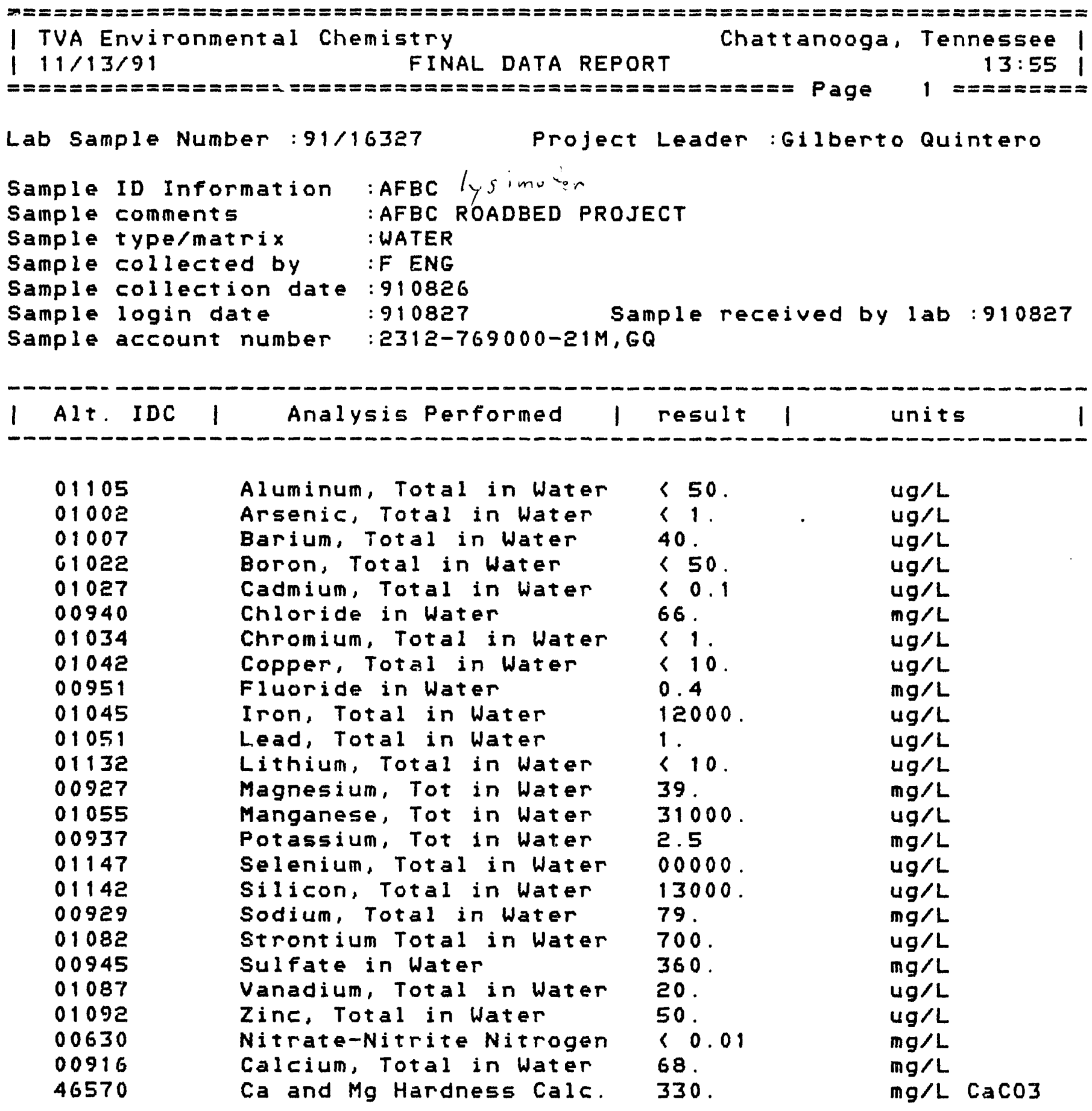



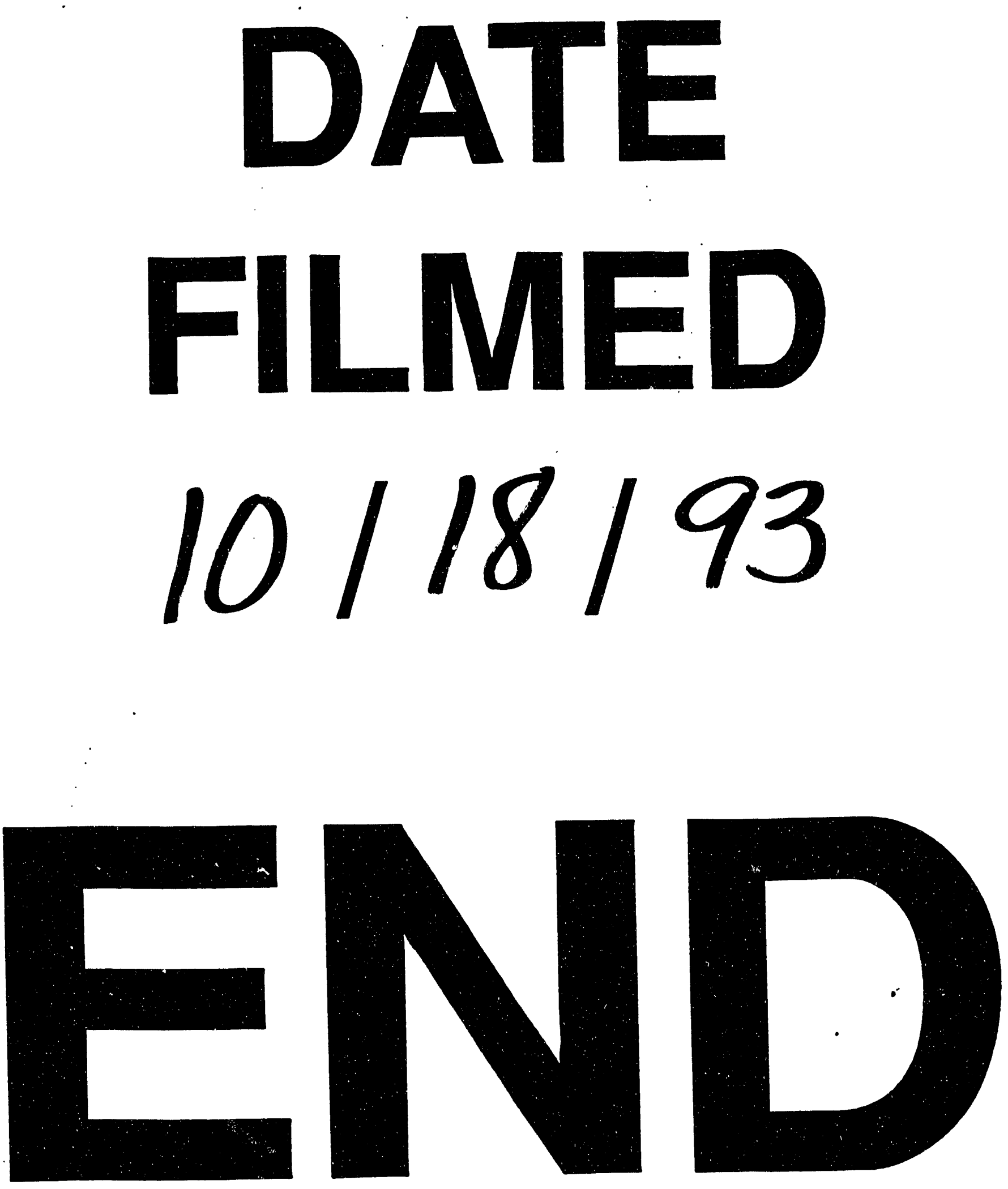
